\title{
Dexmedetomidine Attenuates Neurotoxicity in Developing Rats Induced by Sevoflurane through Upregulating BDNF-TrkB-CREB and Downregulating ProBDNF-P75NRT-RhoA Signaling Pathway
}

\author{
Yunxia Dong $\mathbb{D}^{1},{ }^{1}$ Wei Hong, ${ }^{2}$ Zhiyin Tang, ${ }^{1}$ Yan Gao, ${ }^{1,3}$ Xiuying $\mathrm{Wu}^{1}$ and Hongtao Liu $\mathbb{D}^{1}$ \\ ${ }^{1}$ Department of Anesthesiology, Shengjing Hospital of China Medical University, Shenyang, China \\ ${ }^{2}$ Department of Ultrasound, The Third Affiliated Hospital of Liaoning University of Traditional Chinese Medicine, Shenyang, China \\ ${ }^{3}$ Department of Anesthesiology, The First Affiliated Hospital of Hebei North University, Zhangjiakou, China
}

Correspondence should be addressed to Hongtao Liu; 2895364359@qq.com

Received 18 March 2020; Accepted 23 May 2020; Published 20 June 2020

Academic Editor: Calogero Caruso

Copyright () 2020 Yunxia Dong et al. This is an open access article distributed under the Creative Commons Attribution License, which permits unrestricted use, distribution, and reproduction in any medium, provided the original work is properly cited.

\begin{abstract}
To investigate the mechanism dexmedetomidine in relieving the neurotoxicity of a developing brain induced by sevoflurane. Sprague-Dawley rats, 6 days old, were randomly divided into three groups. Rats in the control group were inhaled with air after injection of normal saline; rats in the sevoflurane group were injected with normal saline and inhaled with $3 \%$ sevoflurane for $2 \mathrm{~h}$ in three consecutive day; rats in the dexmedetomidine group were inhaled with $3 \%$ sevoflurane after intraperitoneal injection of dexmedetomidine $25 \mu \mathrm{g} / \mathrm{kg}$. WB results showed that mBDNF, pTrkB/TrkB, and CREB were significantly decreased in the hippocampus of the sevoflurane group, which are significantly upregulated in the dexmedetomidine group. In the sevoflurane group, proBDNF, P75NRT, and RhoA were significantly increased, which were significantly lower than those in the dexmedetomidine group than those in the sevoflurane group. The expression BDNF was downregulated in the sevoflurane group, while the proBDNF was upregulated in the sevoflurane group. In the Morris water maze test, the escape latency of the sevoflurane group was significantly prolonged. In sevoflurane groups, the number of crossing platform was significantly reduced, the synaptic protein decreased significantly, and this effect was reversed in rats of the dexmedetomidine group. Dexmedetomidine could reduce synaptic plasticity decline in developing rats induced by sevoflurane, through downregulating the proBDNF-p75NTR-RhoA pathway and upregulating BDNF-TrkB-CREB.
\end{abstract}

\section{Introduction}

The human brain has a rapid development period from the last three months of the embryo to the first three years after birth. If molecular structure changes, it will have an effect on the learning and memory ability $[1,2]$. There are many reports stating that long-term, repeated, or high-dose sevoflurane anesthesia in neonatal rats can cause changes in nervous system function and affect the long-term learning and memory and cognitive function [3-5]. The FDA warned that repeated or prolonged (more than $3 \mathrm{~h}$ ) use of anesthetics and sedatives may impair the brain of fetus and children less than 3 years [6].
Dexmedetomidine is a novel, highly selective, highly specific $\alpha 2$ adrenergic receptor agonist which is widely used as a sedative drug in clinic. Recent studies have shown that dexmedetomidine has neuroprotective effects such as on nerves, heart muscles, and kidneys [7-10]. Some studies have demonstrated that dexmedetomidine can attenuate the expression of brain-derived neurotrophic factor (BDNF) in the brain caused by isoflurane, thereby reducing the impact of synaptic plasticity [11-13]. This article explores the mechanism by which dexmedetomidine can alleviate the developmental neurological damage caused by sevoflurane, reduce learning and memory impairment, which paves the foundation for clinical use. 


\section{Materials and Methods}

\subsection{Material}

2.1.1. Animals. A total of 30 Sprague-Dawley (SD) rats (License number SCXK (Liao) 2015-0001), including 10 males and 20 females, weighing 220-250 g, were housed in a light-dark cycle at room temperature $\left(24 \pm 1^{\circ} \mathrm{C}\right)$ for $14: 10$ cycles under free access to water and food. When the females are pregnant, they are kept in separate cages until they give birth naturally. The day of birth of the child was recorded as day 0 (P0). Male rats in each litter were randomly selected for this study in the sixth day (P6).

2.2. Establishment of Sevoflurane Injury Model and Pretreatment with Dexmedetomidine. The animal protocol was approved by the Animal Welfare Ethics Committee of Shengjing Hospital of China Medical University (No. 2019PS010K). Male rats at P6 were randomly selected from each litter. Rats were injected peritoneally with dexmedetomidine $25 \mu \mathrm{g} / \mathrm{kg}$ or the same amount of saline 20 minutes before exposure to sevoflurane or air control. During the experiment, a group of 12 rats were placed in a transparent glass anesthesia box $(30 \mathrm{~cm} * 20 \mathrm{~cm} * 20 \mathrm{~cm})$ for 2 hours per day. An internal heating lamp was used to keep the temperature inside the box at $37^{\circ} \mathrm{C}$. After rats were naturally awakened, the mother rats were placed in cages. On P7 and P8, rats were treated as the same as on P6.

2.3. Western Blot Analysis. The hippocampus tissue was added to the lysate as $1 \mathrm{mg} / 10 \mu \mathrm{L}$, and the hippocampus tissue was cut and sheared. The tissue was thoroughly pulverized, left on ice for $30 \mathrm{~min}$, and centrifuged at $12000 \mathrm{~g}$ for $20 \mathrm{~min}$ at $4^{\circ} \mathrm{C}$, and then, the supernatant was collected. The BCA protein concentration measures were used. Then, proteins were diluted to $40 \mu \mathrm{g} / 10 \mu \mathrm{L}$ and loaded to $10 \mu \mathrm{L}$ on $10 \%$ sodium dodecyl sulfate-polyacrylamide gel electrophoresis (SDS-PAGE). After being separated on SDS-PAGE, proteins were transferred to the PVDF membrane. The PVDF membrane was placed in 5\% skim milk for $4 \mathrm{~h}$ at room temperature, and the membrane was washed 3 times. The primary antibodies were added: anti-BDNF ( $1: 2000$, Abcam, Cambridge, UK), anti-proBDNF (1:500, Alomone Labs, Israel), P75NRT (1:2000, Cell Signaling Technology, USA), TrkB (1:1000, ABclonal, Woburn, MA, USA), p-TrkB ( $1: 1000$, ABclonal, Woburn, MA, USA), CREB ( $1: 1000$, Cell Signaling Technology, USA), anti-RhoA (1:500, Proteintech, China), PSD95 (1:500, Proteintech Biotechnology, Chicago, IL, USA), SYP ( $1: 1000$, Abcam, USA), anti- $\beta$-actin $(1: 500$, Proteintech, China), and GAPDH $(1: 1000$, Cell Signaling Technology, Boston, MA, USA). The membrane was kept overnight at a $4^{\circ} \mathrm{C}$ shaker, and the membrane was washed 3 times with TBST $10 \mathrm{~min}$ each and was added a second antibody (1:5000, Zhongshan Jinqiao Co., Ltd., China). The membrane was stored at room temperature for $90 \mathrm{~min}$ and washed 3 times for $10 \mathrm{~min}$ each. The membrane was added with the chemical luminescent substrate after $5 \mathrm{~min}$, exposed in a C300 illuminator. ImageJ analysis software was used to analyze the results.
2.4. Immunofluorescence Assay. The prepared brain slices were washed with $50 \mathrm{mM}$ PBS for 3 times for $10 \mathrm{~min}$ each, and then, the slices were incubated in $3 \% \mathrm{H}_{2} \mathrm{O}_{2}$ for $30 \mathrm{~min}$ to block endogenous peroxidase activity, followed by immersion in blocking solution for $2 \mathrm{~h}$ and then incubated overnight with BDNF antibody (1:200, Abeam, Cambridge, UK) and proBDNF (1:50, Alomone Labs, Israel). To verify the expression of $\mathrm{BDNF}$ and proBDNF, we used rat antineuronal nuclear antibodies (1:200; Millipore, Billerica, MA, USA) for counterstaining on the same slice. Slices were then incubated with FITC-conjugated goat anti-rabbit secondary antibody (Vector Laboratories Inc., Burlingame, CA, USA) and FITC-conjugated goat anti-mouse secondary antibody (Jackson Immuno Research Laboratories Inc., West) for $2 \mathrm{~h}$. The coverslips were fixed using a fluorescent mounting medium and were captured using a confocal laser scanning microscope of LSM 510 META used to take Fluorescence images.

2.5. Morris Water Maze (MWM) Experiment. Three groups were housed until P28; 10 rats in each group were examined by MWM experiments. The MWM equipment is a circular swimming pool. The escape latency experiment was performed in the first five days, four quadrants per day for five consecutive days, once in each quadrant. The device automatically recorded the time of the rats finding the hidden platform, and after $15 \mathrm{~s}$ being kept on the platform, rats were removed from the platform. If the platform was not found in $90 \mathrm{~s}$, the rat was guided to the platform and stayed for $15 \mathrm{sec}$ onds. After five-day training, the platform was taken out and the rats were allowed to swim freely for $90 \mathrm{~s}$ to record the number of crossing through the platform. All videos, images, and data are recorded and processed using an automatic tracking system (Noldus, Holland).

2.6. Statistical Analysis. Data are expressed as the mean \pm SEM, and multiple comparisons were calculated using oneway ANOVA followed by the Tukey test. GraphPad Prism 6 software (version 6.0; GraphPad Software, Inc.) and SPSS 22.0 software were used to analyze all data. $P<0.05$ was used to consider the difference statistically significantly.

\section{Results}

3.1. Dexmedetomidine Alleviated the Decline in Learning and Memory Ability during Puberty after Sevoflurane Exposure in Developing Rats. On the fifth day, the escape latency of the sevoflurane group was significantly prolonged (Figure 1(a), $P<0.05)$. The number of crossing platforms on the sixth day was significantly reduced (Figure 1(b), $P<0.05$ ). There was no significant difference in swimming speed among the three groups (Figure 1(c), $P<0.05$ ). Multiple exposure to sevoflurane during brain development in rats can affect learning and memory in adolescent rats, while dexmedetomidine can reverse the adverse effects of sevoflurane on learning and memory in the brain. We concluded that the pretreatment of dexmedetomidine can alleviate the long-term learning and memory impairment caused by sevoflurane. 


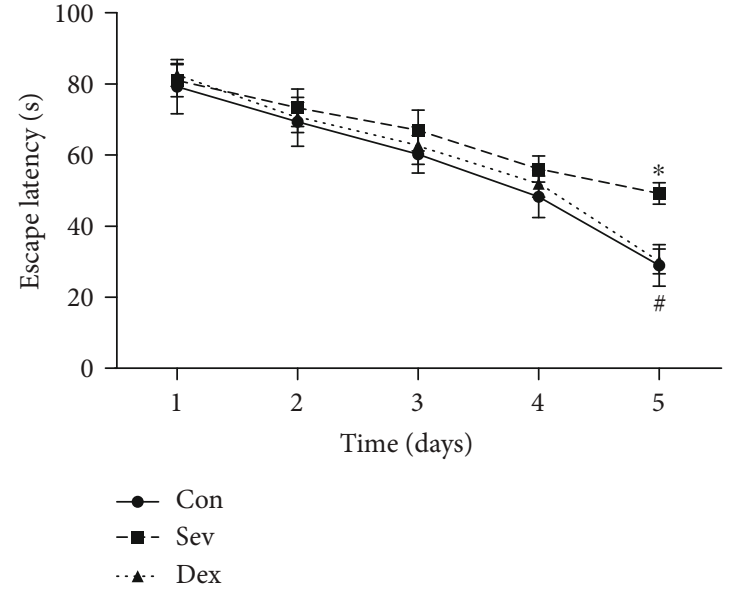

(a)

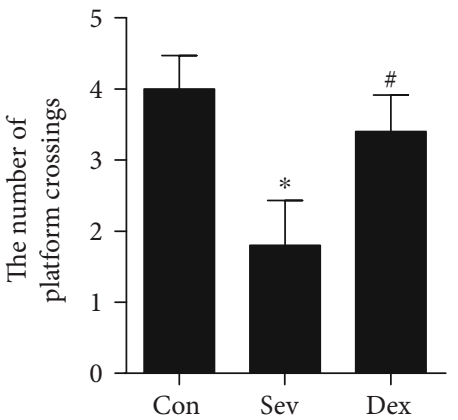

(b)

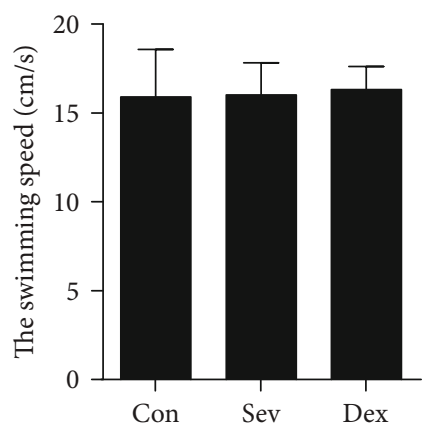

(c)

FiguRE 1: Dexmedetomidine alleviated the decline in learning and memory ability during puberty after sevoflurane exposure in developing rats. (a) The escape latency. (b) The number of crossing platforms on the sixth day. (c) Swimming speed. * Compared with the control group, $P<0.05$. ${ }^{\#}$ Compared with the Sev group, $P<0.05$.

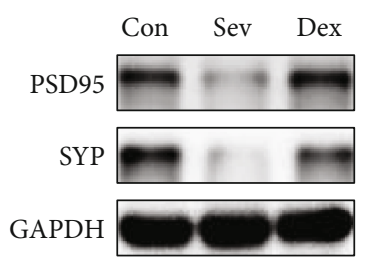

(a)

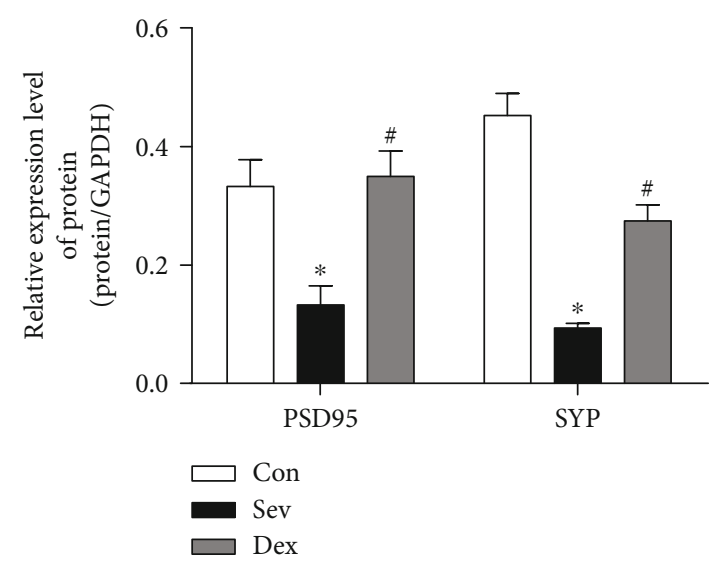

(b)

FIGURE 2: Dexmedetomidine improves hippocampal synaptic protein levels of sevoflurane exposure in developing rats. (a) Western blot band. (b) Bar graph of Western blot. * Compared with the control group, $P<0.05$. ${ }^{*}$ Compared with the Sev group, $P<0.05$.

\subsection{Dexmedetomidine Improves Hippocampal Synaptic} Protein Levels during Puberty after Sevoflurane Exposure in Developing Rats. Although the number of neurons in the brain cannot increase after birth, the density of neurons and the synaptic circuits formed by neurons has been continuously modified [14]. The synaptic density is important for the brain's ability in learning and remember. The proteins representing synaptic density mainly include postsynaptic density protein 95 (PSD95) and synaptophysin (SYP). Our study found that the hippocampal synaptic protein was still lower when the sevoflurane-inhaled rats reached puberty (32 days of birth) (Figure 2). This result was consistent with 

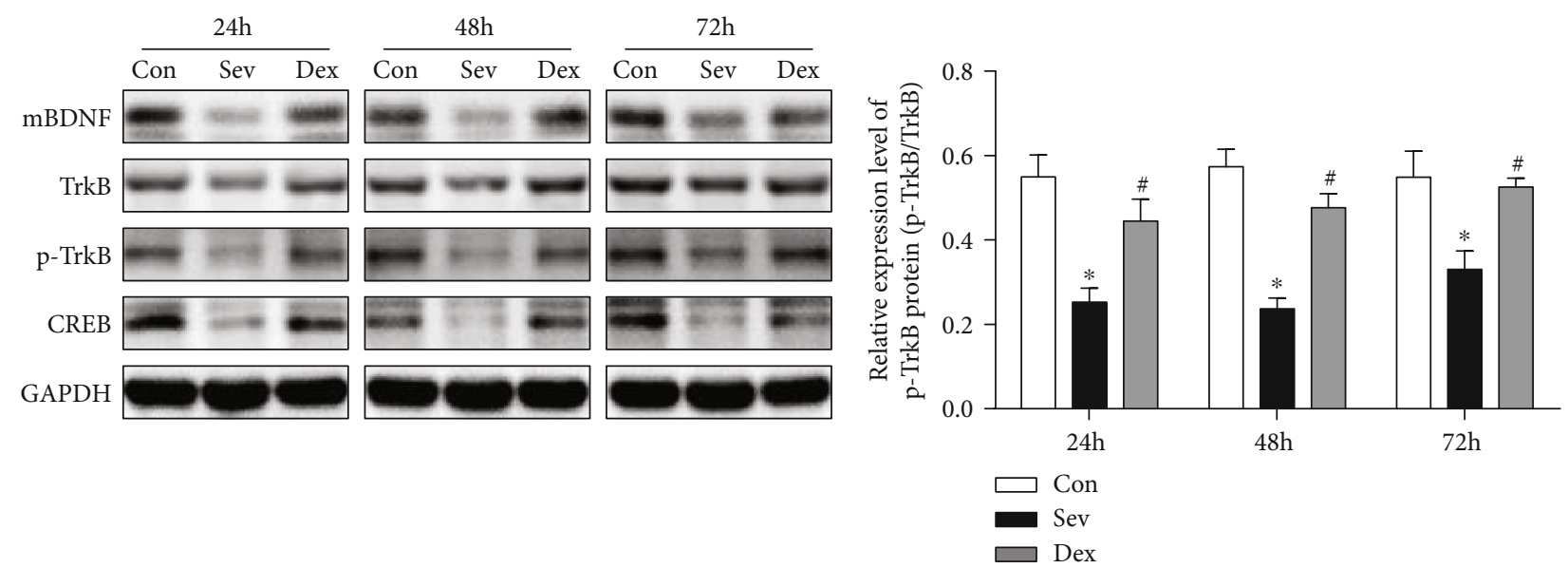

(a)
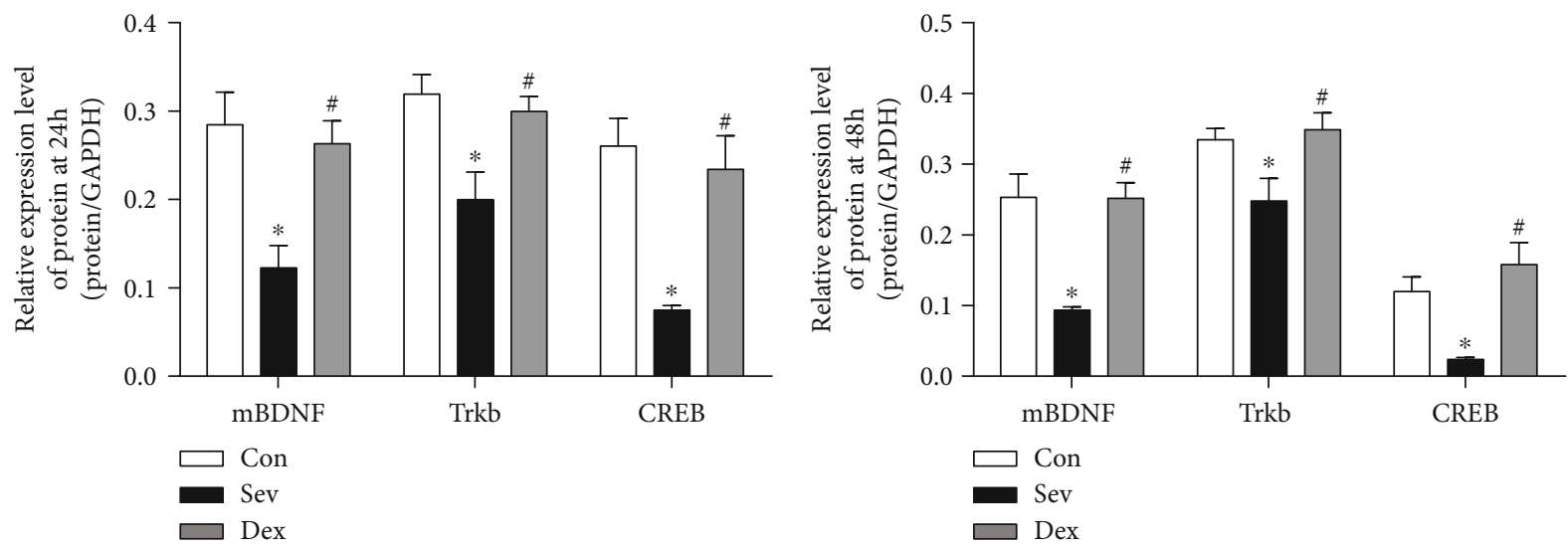

(c)

(d)

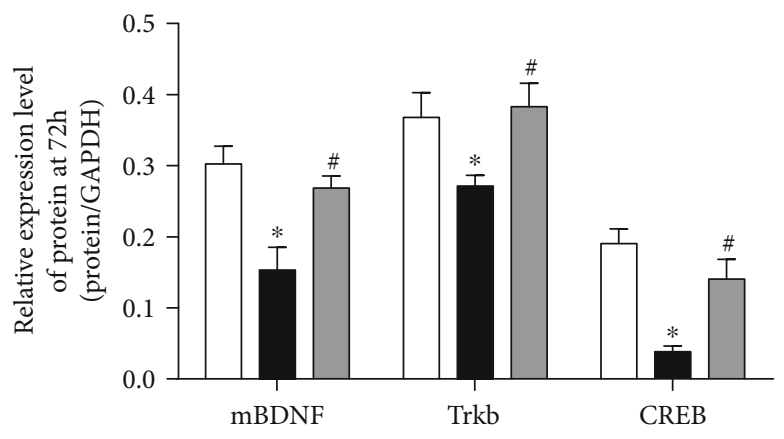

$\square$ Con
Sev
$\square$ Dex

(e)

Figure 3: Continued. 

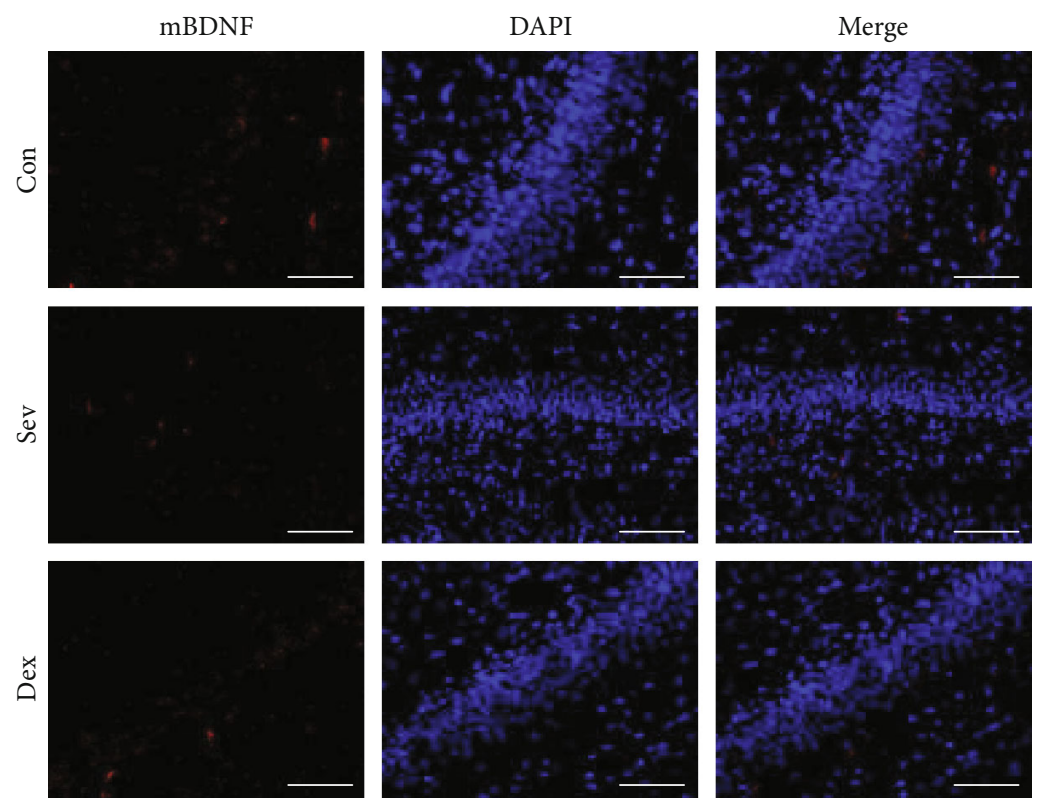

(f)

FIGURE 3: Dexmedetomidine could increase the level of mBDNF and relieve the inhibition of the BDNF-TrkB-CREB pathway caused by sevoflurane in the hippocampus. (a) Western blot band. (b-e) Bar graph of Western blot. (f) Immunofluorescence of mBDNF (scale bar $=50 \mu \mathrm{m})$. ${ }^{*}$ Compared with the control group, $P<0.05$; ${ }^{\#}$ Compared with the Sev group, $P<0.05$.

our previous research and other literature reports [15-18]. We found that preinjection of dexmedetomidine before sevoflurane exposure can alleviate the decline of long-term hippocampal synaptic protein SYP and PSD95 after sevoflurane anesthesia, suggesting that the protection of dexmedetomidine can last for long term.

\subsection{The Pretreatment of Dexmedetomidine Could Increase the} Level of Hippocampal Mature Brain-Derived Neurotrophic Factor mBDNF in Rats Exposed by Sevoflurane Increase. The lack of BDNF can significantly reduce synaptic plasticity, which in turn affects synaptic development and growth, leading to decreased learning and memory. Our study found that BDNF, TrkB, and CREB levels in the hippocampus were significantly decreased after sevoflurane exposed in developing rats $(P<0.05)$. Pretreatment with dexmedetomidine significantly ameliorated the decreasing of hippocampal mBDNF, p-TrkB, TrkB, and CREB protein (Figures 3(a)-3(e)). Immunofluorescence results showed that $\mathrm{mBDNF}$ expression was lowest in the hippocampal CA1 region in the sevoflurane group, while $\mathrm{mBDNF}$ expression in the dexmedetomidine group was significantly higher than that in the sevoflurane group (Figure 3(f)). It indicated that dexmedetomidine increased the expression of mBDNF in the brain, thus activating the mBDNF-TrkB-CREB pathway and ameliorating the abnormal expression of mBDNF caused by sevoflurane.

\subsection{Dexmedetomidine Inhibits proBDNF-P75NRT-RhoA} Signaling Pathway Caused by Sevoflurane in the Hippocampus. The pretreatment of dexmedetomidine could relieve the obstacles of proBDNF cleavage in the hippocampus caused by sevoflurane exposure, which could reduce the ratio of proBDNF/mBDNF, and inhibit the proBDNF-
P75NRT-RhoA signaling pathway. The BDNF precursor, proBDNF, is proteolytically cleaved into a mature form of BDNF. Many reports have confirmed proBDNF has intrinsic biological function, which is completely opposite to mBDNF [19]. ProBDNF can preferentially bind to p75NTR, which in turn activates RhoA, promotes apoptosis, and collapses of nerve cone, thus affecting synaptic plasticity [20-22]. We have shown that the proBDNF increased in the hippocampus after sevoflurane exposure, especially the ratio of proBDNF/mBDNF which is significantly increased. But dexmedetomidine can reduce the expression of proBDNF in the hippocampus and decrease the ratio of proBDNF/mBDNF. At the same time, P75NRT and RHOA increased significantly after sevoflurane exposure, and the expression of P75NRT and RHOA became normal after pretreatment of dexmedetomidine (Figure 4).

\section{Discussion}

A recent high-quality clinical randomized controlled trial reported that patients in an intensive care unit, pretreated with low-dose dexmedetomidine, could significantly reduce the incidence of delirium within $7 \mathrm{~d}$ after surgery and reduce postoperative patients' anxiety [23]. Basic experimental studies have found that dexmedetomidine can prevent learning and memory impairment caused by different brain injury models [24-26]. In recent years, some teams have confirmed that dexmedetomidine can counteract neuropathy or brain function changes in puberty caused by sevoflurane, a commonly used anesthetic drug in children, by reducing oxidative stress and reducing apoptosis $[27,28]$. In our study, dexmedetomidine has a neuroprotective effect on the brain after sevoflurane exposure, and this protection can continue 

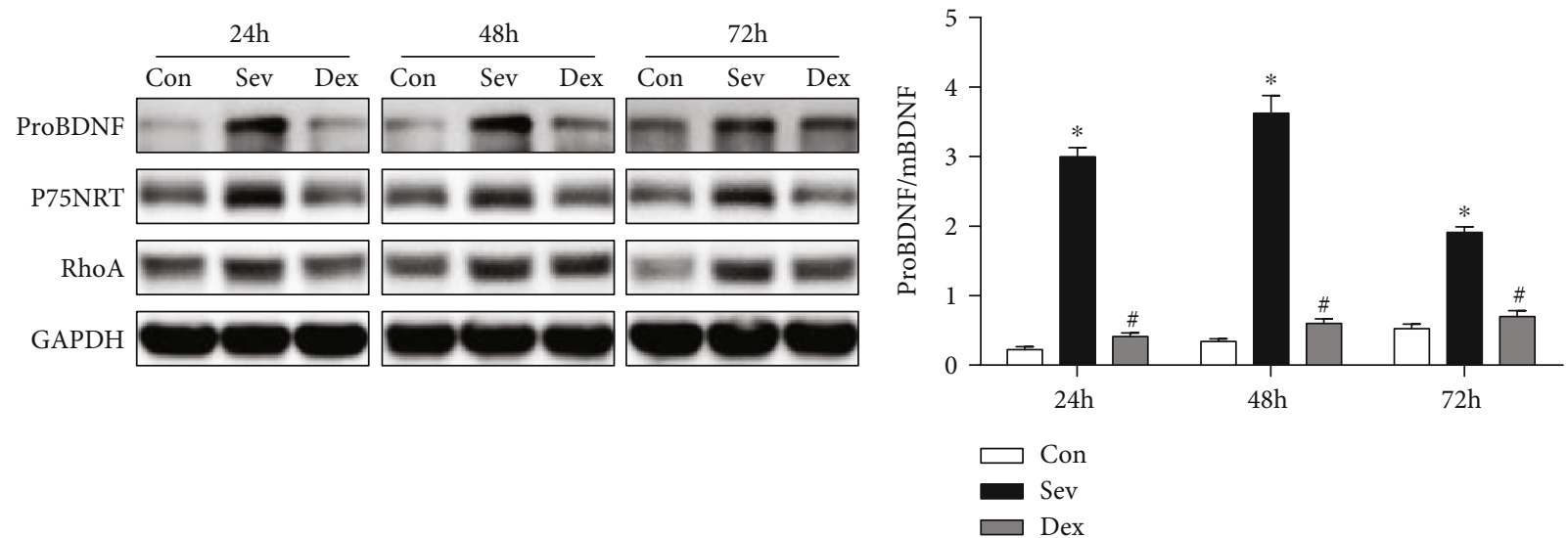

(a)

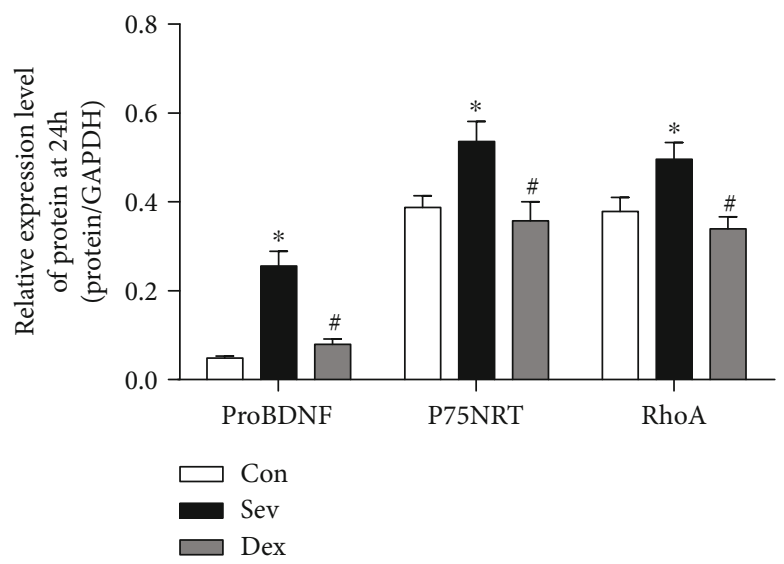

(c) (b)

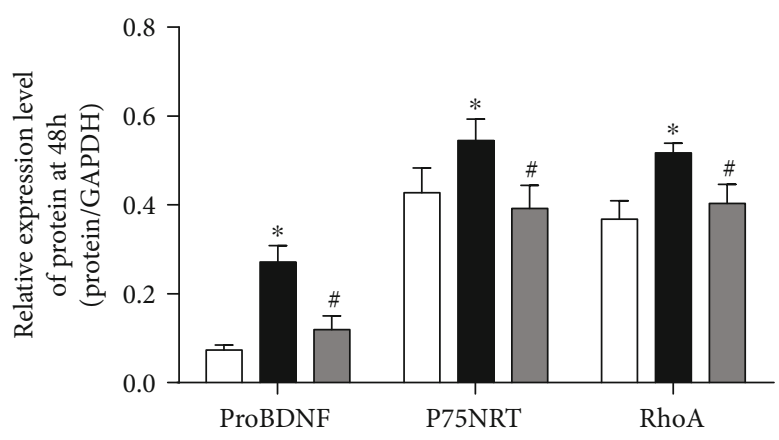

$\square$ Con

- Sev

$\square$ Dex

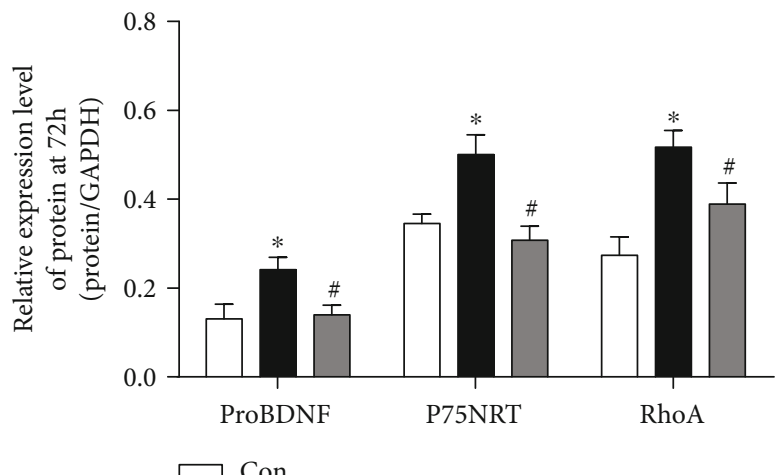

$\square$ Con

- Sev

(e)

Figure 4: Continued. 

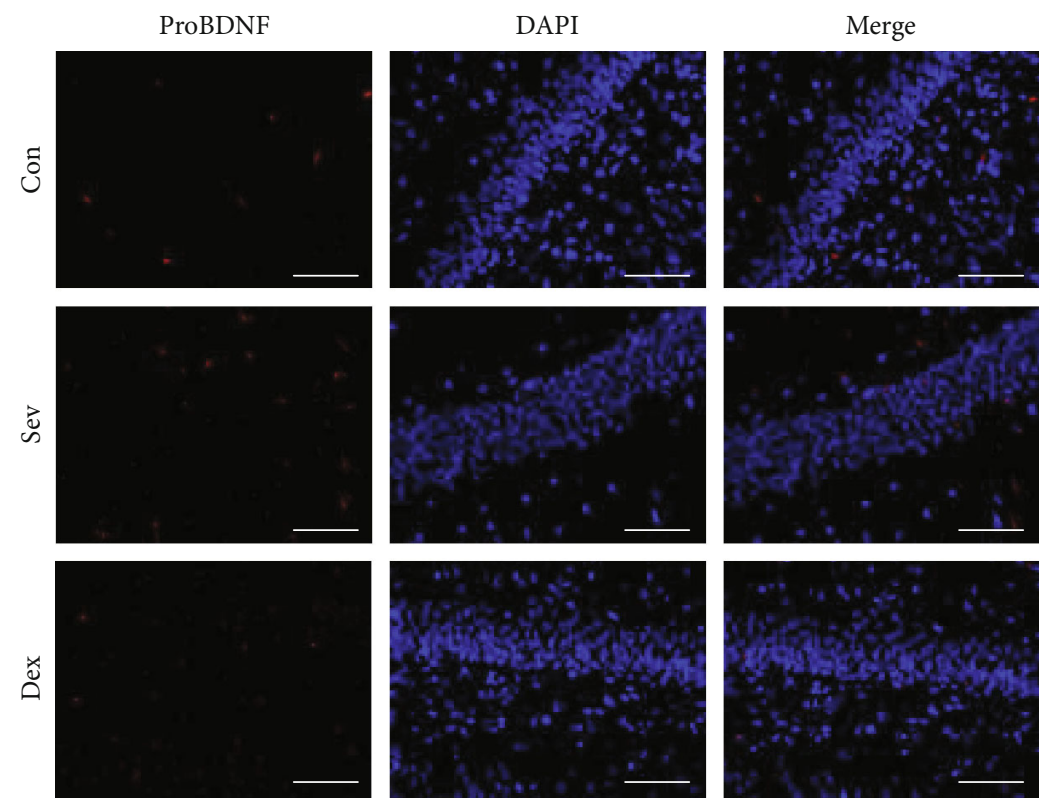

(f)

FIgURE 4: Dexmedetomidine could decrease the level of proBDNF and restore the ratio of proBDNF/mBDNF and alleviates activation of the proBDNF-P75NRT-RHOA pathway after sevoflurane. (a) Western blot band. (b) ProBDNF/mBDNF. (c-e) Bar graph of Western blot. (f) Immunofluorescence of proBDNF (scale bar $=50 \mu \mathrm{m}$ ). ${ }^{*}$ Compare with the control group, $P<0.05$; ${ }^{\#}$ Compared with the Sev group, $P<0.05$.

until puberty, relieving the reduction of learning and memory ability in puberty caused by sevoflurane. Dexmedetomidine provided neuroprotection against sevoflurane-induced neuroapoptosis in a dose-dependent manner. However, several reports describe how dexmedetomidine lacks neurotoxicity even at extremely high doses such as $75 \mu \mathrm{g} / \mathrm{kg}$. Pretreatment once with dexmedetomidine $(75 \mu \mathrm{g} / \mathrm{kg})$ provides a similar effect to that of applying $25 \mu \mathrm{g} / \mathrm{kg}[29,30]$. However, it has been reported recently that large doses of dexmedetomidine combined with sevoflurane may cause respiratory depression, carbon dioxide retention, asphyxia, death, and other adverse reactions, which may be due to different conditions of model preparation [28]. Therefore, we chose $25 \mu \mathrm{g} / \mathrm{kg}$ for intraperitoneal administration at $20 \mathrm{~min}$ before sevoflurane exposure for three consecutive days. There were no death of rats and adverse effects of respiratory depression during the experiment. On the 20th day after the experiment, the protection of learning and memory ability can be observed and synaptic proteins were significantly attenuated in the hippocampal region of developing rats. Thus, $25 \mu \mathrm{g} / \mathrm{kg}$ of dexmedetomidine was sufficient to induce neuroprotection.

As many studies have shown that the main reason for the changes in learning and memory ability caused by inhaled anesthetics is mainly due to the prominent plasticity of brain neurons such as synaptic density or reticular formation rather than neuronal abnormalities caused by apoptosis or oxidative stress $[31,32]$. BDNF plays an important role in the regulation of synaptic plasticity, which is the basis of the brain's ability in learning and remembering. Thus, the main cause of inhaled anesthetics leading to brain learning and memory is the decline of BDNF in the brain $[33,34]$.
Our previous study found that dexmedetomidine reversed the abnormal expression of NR2A, EAAT1, and NR2B caused by sevoflurane and increased MLP2 expression, which was through the BMP/SMAD signaling pathway $[25,35]$. We believe that dexmedetomidine protection on the brain during development after sevoflurane exposure is not only through the antiapoptosis and antioxidative stress. In this study, we found that the level of mBDNF in the hippocampus decreased after multiple exposure with sevoflurane and the level of BDNF-TrkB-CREB-related proteins was downregulated, which is the key pathway that determines synaptic plasticity $[36,37]$. We clearly found that the levels TrkB and CREB proteins decreased. This recent decline in protein after modeling could cause the reduction of PSD95 and SYP levels in long term. PSD95 and SYP, markers of presynaptic and postsynaptic components, are involved in synapse formation and reconstruction [38-42]. Decreased protein expression means the loss of synapses, hippocampal synaptic plasticity decreases after multiple anesthesia with sevoflurane, and this effect can continue to puberty, accompanied by decreased synaptic protein levels during adolescence and decline of learning and memory function. After the treatment of dexmedetomidine, the decrease of mBDNF, TrkB, and CREB protein after sevoflurane exposure was alleviated, synaptic proteins were showed to return to normal, and thus, the learning and memory ability was improved at $28 \mathrm{~d}$ after modeling. These results suggested that dexmedetomidine may restore abnormalities in long-term synaptic protein expression and behavioral abnormalities by improving the BDNF-TrkB protective pathway.

At the same time, we found that the proBDNF in the hippocampus increased after sevoflurane exposure; especially, 
the ratio of proBDNF/mBDNF increased significantly. This result is consistent with the head team's report that isoflurane could increase the proBDNF in vitro [43]. The cleavage of proBDNF is reduced leading to $\mathrm{mBDNF}$ decreasing; thus, proBDNF is relatively more abundant than normal condition. Previous literatures have shown that there is a reverse regulation of increased proBDNF in different pathological models. ProBDNF binds to P75NRT and activates the downstream RhoA-Rock pathway, causing cascades of neurite outgrowth and filopodial growth cones by activating RhoA [44-47]. The recently pathway protein changes could affect the long-term synaptic protein changes and behavioral changes. Our observations suggest that proBDNF collapses neurite outgrowth and filopodial growth cones by activating RhoA through the p75NTR signaling pathway. After pretreatment of dexmedetomidine, we found that the ratio of proBDNF/mBDNF was significantly decreased, the pathway proteins were significantly decreased, and this effect continued into puberty. Therefore, dexmedetomidine can alleviate the increase of proBDNF after sevoflurane exposure and decrease RhoA protein. It may be the protective effect of the developmental brain.

There is a limit in this experiment. We did not use pathway agonists and inhibitors or knockout rats to observe the specific targeting of dexmedetomidine but only detected the protein expression changes to speculate the mechanism of action of dexmedetomidine.

These data demonstrated that pretreatment with dexmedetomidine may offer a strategy for the protection of the neonatal brain during sevoflurane anesthesia.

We first reported that the brain protection of dexmedetomidine may be associated with restoring the ratio of proBDNF/mBDNF after sevoflurane exposure. Then, the hippocampal synaptic proteins were corrected during puberty, and memory impairment was repaired after sevoflurane exposure. Dexmedetomidine could alleviate sevoflurane-induced proBDNF aggregation and thus increase mature BDNF and activate the BDNF-directed TrkB-CREB pathway. Dexmedetomidine also reduces proBDNF levels, thus alleviates sevoflurane activation of the proBDNF-P75NRT-RHOA pathway, thereby reduces the effects of sevoflurane exposure on synaptic plasticity, and reduces the decline in learning and memory caused by sevoflurane.

\section{Data Availability}

The datasets used and/or analyzed during the current study are available from the corresponding author on reasonable request.

\section{Conflicts of Interest}

The authors declare that they have no conflicts of interest.

\section{References}

[1] F. Buttelmann and J. Karbach, "Development and plasticity of cognitive flexibility in early and middle childhood," Frontiers in Psychology, vol. 8, article 1040, 2017.
[2] J. A. Stogsdill and C. Eroglu, "The interplay between neurons and glia in synapse development and plasticity," Current Opinion in Neurobiology, vol. 42, pp. 1-8, 2017.

[3] B. Zhou, L. Chen, P. Liao et al., "Astroglial dysfunctions drive aberrant synaptogenesis and social behavioral deficits in mice with neonatal exposure to lengthy general anesthesia," PLoS Biology, vol. 17, no. 8, article e3000086, 2019.

[4] K. E. M. Lee, T. G. Diacovo, J. Calderon, M. W. Byrne, and C. Ing, "Outcomes research in vulnerable pediatric populations," Journal of Neurosurgical Anesthesiology, vol. 31, no. 1, pp. 140-143, 2019.

[5] C. S. Houck and A. E. Vinson, "Anaesthetic considerations for surgery in newborns," Archives of Disease in Childhood - Fetal and Neonatal Edition, vol. 102, no. 4, pp. F359-F363, 2017.

[6] D. B. Andropoulos and M. F. Greene, "Anesthesia and developing brains - implications of the FDA warning," New England Journal of Medicine, vol. 376, no. 10, pp. 905-907, 2017.

[7] B. Subramaniam, P. Shankar, S. Shaefi et al., "Effect of intravenous acetaminophen vs placebo combined with propofol or dexmedetomidine on postoperative delirium among older patients following cardiac surgery: the DEXACET randomized clinical trial," JAMA, vol. 321, no. 7, pp. 686-696, 2019.

[8] C. Liu, Q. Fu, R. Mu et al., "Dexmedetomidine alleviates cerebral ischemia-reperfusion injury by inhibiting endoplasmic reticulum stress dependent apoptosis through the PERKCHOP- Caspase-11 pathway," Brain Research, vol. 1701, pp. 246-254, 2018.

[9] L. He, S. Hao, Y. Wang et al., "Dexmedetomidine preconditioning attenuates ischemia/reperfusion injury in isolated rat hearts with endothelial dysfunction," Biomedicine \& Pharmacotherapy, vol. 114, article 108837, 2019.

[10] J. Ma, Q. Chen, J. Li et al., "Dexmedetomidine-mediated prevention of renal ischemia-reperfusion injury depends in part on cholinergic anti-inflammatory mechanisms," Anesthesia \& Analgesia, vol. 130, no. 4, pp. 1054-1062, 2020.

[11] Y. Ge, Q. Li, Y. Nie et al., "Dexmedetomidine improves cognition after carotid endarterectomy by inhibiting cerebral inflammation and enhancing brain-derived neurotrophic factor expression," Journal of International Medical Research, vol. 47, no. 6, pp. 2471-2482, 2019.

[12] Y. Tu, Y. Liang, Y. Xiao et al., "Dexmedetomidine attenuates the neurotoxicity of propofol toward primary hippocampal neurons in vitro via Erk1/2/CREB/BDNF signaling pathways," Drug Design, Development and Therapy, vol. 13, pp. 695-706, 2019.

[13] Y. Wang, A. Jia, and W. Ma, "Dexmedetomidine attenuates the toxicity of $\beta$-amyloid on neurons and astrocytes by increasing BDNF production under the regulation of HDAC2 and HDAC5," Molecular Medicine Reports, vol. 19, no. 1, pp. 533-540, 2018.

[14] K. P. Berry and E. Nedivi, "Spine dynamics: are they all the same?," Neuron, vol. 96, no. 1, pp. 43-55, 2017.

[15] F. Yang, Y. Shan, Z. Tang et al., "The neuroprotective effect of hemin and the related mechanism in sevoflurane exposed neonatal rats," Frontiers in Neuroscience, vol. 13, p. 537, 2019.

[16] X. Ju, Y. Jang, J. Y. Heo et al., “Anesthesia affects excitatory/inhibitory synapses during the critical synaptogenic period in the hippocampus of young mice: Importance of sex as a biological variable," Neurotoxicology, vol. 70, pp. 146-153, 2019. 
[17] H. Li, C. L. Dai, J. H. Gu et al., "Intranasal administration of insulin reduces chronic behavioral abnormality and neuronal apoptosis induced by general anesthesia in neonatal mice," Frontiers in Neuroscience, vol. 13, p. 706, 2019.

[18] G. Li, J.'. du, L. Wang, and X. Shi, “Developmental neurotoxicity in the context of multiple sevoflurane exposures: potential role of histone deacetylase 6," Neurotoxicology and Teratology, vol. 74, article 106813, 2019.

[19] P. T. Pang, H. K. Teng, E. Zaitsev et al., "Cleavage of proBDNF by tPA/plasmin is essential for long-term hippocampal plasticity," Science, vol. 306, no. 5695, pp. 487-491, 2004.

[20] K. Saadipour, M. Yang, Y. Lim et al., "Amyloid beta ${ }_{1-42}\left(\mathrm{~A} \beta_{42}\right)$ up-regulates the expression of sortilin via the $\mathrm{p} 75^{\mathrm{NTR}} / \mathrm{RhoA}$ signaling pathway," Journal of Neurochemistry, vol. 127, no. 2, pp. 152-162, 2013.

[21] Y. Sun, Y. Lim, F. Li et al., "ProBDNF collapses neurite outgrowth of primary neurons by activating RhoA," PLoS One, vol. 7, no. 4, article e35883, 2012.

[22] L. L. Orefice, C. C. Shih, H. Xu, E. G. Waterhouse, and B. Xu, "Control of spine maturation and pruning through proBDNF synthesized and released in dendrites," Molecular and Cellular Neuroscience, vol. 71, pp. 66-79, 2016.

[23] X. Su, Z. T. Meng, X. H. Wu et al., "Dexmedetomidine for prevention of delirium in elderly patients after non- cardiac surgery: a randomised, double-blind, placebo-controlled trial," The Lancet, vol. 388, no. 10054, pp. 1893-1902, 2016.

[24] X. Gao, F. Z. Zhuang, S. J. Qin et al., "Dexmedetomidine protects against learning and memory impairments caused by electroconvulsive shock in depressed rats: involvement of the NMDA receptor subunit 2B (NR2B)-ERK signaling pathway," Psychiatry Research, vol. 243, pp. 446-452, 2016.

[25] Y. Shan, F. Yang, Z. Tang et al., "Dexmedetomidine ameliorates the neurotoxicity of sevoflurane on the immature brain through the BMP/SMAD signaling pathway," Frontiers in Neuroscience, vol. 12, p. 964, 2018.

[26] L. Radek, R. E. Kallionpää, M. Karvonen et al., "Dreaming and awareness during dexmedetomidine- and propofol-induced unresponsiveness," British Journal of Anaesthesia, vol. 121, no. 1, pp. 260-269, 2018.

[27] C. Yi, Z. Fu, and X. Luo, "Dexmedetomidine on autophagy of hippocampal neurons in aged rats under sevoflurane anesthesia," Experimental and Therapeutic Medicine, vol. 16, no. 2, pp. 837-841, 2018.

[28] J. F. Perez-Zoghbi, W. Zhu, M. R. Grafe, and A. M. Brambrink, "Dexmedetomidine-mediated neuroprotection against sevoflurane-induced neurotoxicity extends to several brain regions in neonatal rats," British Journal of Anaesthesia, vol. 119, no. 3, pp. 506-516, 2017.

[29] R. D. Sanders, J. Xu, Y. Shu et al., "Dexmedetomidine attenuates isoflurane-induced neurocognitive impairment in neonatal rats," Anesthesiology, vol. 110, no. 5, pp. 1077-1085, 2009.

[30] Z. Liao, D. Cao, X. Han et al., "Both JNK and P38 MAPK pathways participate in the protection by dexmedetomidine against isoflurane-induced neuroapoptosis in the hippocampus of neonatal rats," Brain Research Bulletin, vol. 107, pp. 69-78, 2014.

[31] H. Lu, N. Liufu, Y. Dong et al., "Sevoflurane acts on ubiquitination-proteasome pathway to reduce postsynaptic density 95 protein levels in young mice," Anesthesiology, vol. 127, no. 6, pp. 961-975, 2017.
[32] M. C. Alvarado, K. L. Murphy, and M. G. Baxter, "Visual recognition memory is impaired in rhesus monkeys repeatedly exposed to sevoflurane in infancy," British Journal of Anaesthesia, vol. 119, no. 3, pp. 517-523, 2017.

[33] B. Liu, G. Ou, Y. Chen, and J. Zhang, "Inhibition of protein tyrosine phosphatase $1 \mathrm{~B}$ protects against sevoflurane- induced neurotoxicity mediated by ER stress in developing brain," Brain Research Bulletin, vol. 146, pp. 2839, 2019.

[34] Z. Wu, X. Li, Y. Zhang, D. Tong, L. Wang, and P. Zhao, "Effects of sevoflurane exposure during mid-pregnancy on learning and memory in offspring rats: beneficial effects of maternal exercise," Frontiers in Cellular Neuroscience, vol. 12, p. 122, 2018.

[35] X. Wang, Y. Shan, Z. Tang, L. Gao, and H. Liu, "Neuroprotective effects of dexmedetomidine against isoflurane-induced neuronal injury via glutamate regulation in neonatal rats," Drug Design, Development and Therapy, vol. 13, pp. 153-160, 2019.

[36] J. Kim, S. Lee, B. R. Choi et al., "Sulforaphane epigenetically enhances neuronal BDNF expression and TrkB signaling pathways," Molecular Nutrition \& Food Research, vol. 61, no. 2, 2017.

[37] D. Panja and C. R. Bramham, "BDNF mechanisms in late LTP formation: a synthesis and breakdown," Neuropharmacology, vol. 76, pp. 664-676, 2014.

[38] S. Yadav, J. A. Oses-Prieto, C. J. Peters et al., “TAOK2 kinase mediates PSD95 stability and dendritic spine maturation through septin7 phosphorylation," Neuron, vol. 93, no. 2, pp. 379-393, 2017.

[39] Y. Lu, G. Sun, F. Yang et al., "Baicalin regulates depression behavior in mice exposed to chronic mild stress via the Rac/LIMK/cofilin pathway," Biomedicine \& Pharmacotherapy, vol. 116, article 109054, 2019.

[40] X. Xiao, X. Xu, F. Li, G. Xie, and T. Zhang, "Anti-inflammatory treatment with $\beta$-asarone improves impairments in social interaction and cognition in MK-801 treated mice," Brain Research Bulletin, vol. 150, pp. 150-159, 2019.

[41] Q. Ke, R. Li, L. Cai, S. D. Wu, and C. M. Li, "Ro41-5253, a selective antagonist of retinoic acid receptor $\alpha$, ameliorates chronic unpredictable mild stress-induced depressive-like behaviors in rats: Involvement of regulating HPA axis and improving hippocampal neuronal deficits," Brain Research Bulletin, vol. 146, pp. 302-309, 2019.

[42] X. Y. Wang, H. R. Zhou, S. Wang et al., "NR2B-Tyr phosphorylation regulates synaptic plasticity in central sensitization in a chronic migraine rat model," The Journal of Headache and Pain, vol. 19, no. 1, p. 102, 2018.

[43] B. P. Head, H. H. Patel, I. R. Niesman, J. C. Drummond, D. M. Roth, and P. M. Patel, "Inhibition of p75 neurotrophin receptor attenuates isoflurane-mediated neuronal apoptosis in the neonatal central nervous system," Anesthesiology, vol. 110, no. 4, pp. 813-825, 2009.

[44] R. He, W. Han, X. Song, X. Tang, L. Cheng, and L. Jiang, "Effect of fasudil on cognitive function following status convulsion in rats," Molecular Medicine Reports, vol. 16, no. 1, pp. 119-126, 2017.

[45] L. Li and B. Liu, "ROCK inhibitor Y-27632 protects rats against cerebral ischemia/reperfusion-induced behavioral deficits and hippocampal damage," Molecular Medicine Reports, vol. 20, no. 4, pp. 3395-3405, 2019. 
[46] C. Xu, X. Wu, M. Lu et al., "Protein tyrosine phosphatase 11 acts through RhoA/ROCK to regulate eosinophil accumulation in the allergic airway," The FASEB Journal, vol. 33, no. 11, pp. 11706-11720, 2019.

[47] M. Zhao, Y. Tang, P. J. Ernst et al., "Enhancing the engraftment of human induced pluripotent stem cell-derived cardiomyocytes via a transient inhibition of Rho kinase activity," Journal of Visualized Experiments, no. 149, p. e59452, 2019. 